\title{
A Novel Mating Approach for Genetic Algorithms
}

\author{
Severino F. Galan \\ Ole J. Mengshoel \\ Rafael Pinter
}

July 24, 2012

CMU-SV-12-005

\section{Carnegie MellonUniversity SiliconValley}

Silicon Valley Campus 